\title{
Interleukin-13 peptide kinoid vaccination attenuates allergic inflammation in a mouse model of asthma
}

\author{
QIUPING JING ${ }^{1}$, TAO YIN $^{1}$, YANG WAN ${ }^{1}$, HUASHAN SHI $^{1}$, SHUNTAO LUO $^{1}$, MENG LI $^{1}$, \\ HAILONG ZHANG ${ }^{1}$, HONG HE ${ }^{1}$, SHIJIA LIU ${ }^{2}$, HAOYU LI $^{2}$, YUQUAN WEI ${ }^{1}$ and LI YANG ${ }^{1}$ \\ ${ }^{1}$ State Key Laboratory of Biotherapy and Cancer Center, West China Hospital, West China Medical \\ School, and ${ }^{2}$ School of Life Science, Sichuan University, Chengdu, Sichuan, P.R. China
}

Received March 6, 2012; Accepted May 7, 2012

DOI: $10.3892 /$ ijmm.2012.1036

\begin{abstract}
Asthma is an atopic disorder with increasing frequency and severity in developed nations. Interleukin-13 (IL-13) is one of the most critical mediators of asthma pathology. In the present study, we developed a vaccine comprised of a keyhole limpet hemocyanin-mIL-13 heterocomplex immunogen to persistently neutralize excessive endogenous IL-13. Our results showed that the IL-13 peptide kinoid vaccine could induce sustained and high titer of IL-13-specific IgG when using aluminum hydroxide as an adjuvant, and could suppress the accumulation of eosinophils as well as IL-13 levels in bronchoalveolar lavage fluid (BALF). In addition, total $\operatorname{IgE}$ and ovalbumin (OVA)-specific IgE in serum were significantly inhibited. This study also showed that vaccination could prevent airway inflammation and epithelial cell proliferation with goblet cell hyperplasia in a mouse model of acute asthma. In summary, our findings suggest that the IL-13 peptide kinoid can serve as an innovative and effective vaccine against asthma.
\end{abstract}

\section{Introduction}

Asthma is a group of complex diseases associated with allergic airway inflammation, airway hyperresponsiveness (AHR) to a

Correspondence to: Professor Li Yang, State Key Laboratory of Biotherapy and Cancer Center, West China Hospital, Sichuan University, Ke-yuan Road 4, Chengdu, Sichuan 610041, P.R. China E-mail: yl.tracy73@gmail.com

Abbreviations: IL-13, interleukin-13; BALF, bronchoalveolar lavage fluids; OVA, ovalbumin; AHR, airway hyperresponsiveness; IgE, immunoglobulin $\mathrm{E}$; IgG, immunoglobulin G; mAb, monoclonal antibody; MMP-9, matrix metalloproteinase-9; KLH, keyhole limpet hemocyanin; ELISA, enzyme-linked immunosorbent assay; VEGF, vascular endothelial growth factor; TNF, tumor necrosis factor; IL-4, interleukin-4; IFN- $\gamma$, immunoreactive fibronectin- $\gamma$; IFN- $\alpha$, immunoreactive fibronectin- $\alpha$; H\&E, hematoxylin and eosin; PAS, periodic acid shiff; CFA/IFA, complete/incomplete Freund's adjuvant; NMPS, neutral mucopolysaccharide; AMPS, acid mucopolysaccharide

Key words: asthma, interleukin-13, vaccine, kinoid variety of specific and nonspecific stimuli, chronic pulmonary eosinophil infiltration, increased serum immunoglobulin $\mathrm{E}$ (IgE), excessive mucus secretion and other features. At present, there is no cure for asthma. Moreover, the frequency and severity of asthma have increased steadily in developed nations (1). The pathophysiology of asthma is thought to be mediated by activated $\mathrm{CD} 4^{+} \mathrm{T}$ lymphocytes, which produce type 2 cytokines interleukin (IL)-4, IL-5, IL-9 and IL-13 $(2,3)$. Studies have revealed that IL-13 is the most important mediator of asthma. Daily administration of recombinant IL-13 (rIL-13) to the airways of unimmunized mice could induce AHR, whereas blockade of IL-13 by a soluble IL-13 $\alpha 2$ IgGFc fusion protein (sIL-13 $\alpha 2-\mathrm{Fc}$ ) could result in a complete reversal of such allergen-induced AHR $(1,4)$. In addition, therapeutic dosing with an anti-IL-13 monoclonal antibody (mAb) could effectively reverse established AHR, and limit asthmatic responses in a chronic mouse model of persistent asthma (5). Moreover, neutralization of IL-13 could inhibit the production of multiple cytokines, chemokines, and matrix metalloproteinase-9 (MMP-9) (5). It has become increasingly clear that IL-13 is a novel therapeutic target for the treatment of asthma.

A variety of monoclonal antibodies and soluble receptors blocking IL-13 have been developed for the treatment of asthma, and some of them are currently being evaluated in clinical development (6). These approaches are mainly based on the development and administration of functional recombinant protein antagonists that neutralize the extracellular IL-13 function or block IL-13 signaling in target cells. Although successful, these strategies also possess many disadvantages, including difficulties in manufacturing active recombinant protein, high-dose requirements, high costs for manufactures and consumers, and the probable need for lifetime treatment of the patient. Due to their relatively short half-lives, recombinant proteins must be administered repeatedly by injection once to several times a day (7). Moreover, these approaches may have the risk of potential side effects, such as infusion reactions and the production of antibodies to the therapeutic agent (8). A peptide of IL-13 conjugated to truncated hepatitis B core antigen has been developed and utilized in mouse asthma models (9). The mice immunized with IL-13 peptidebased vaccine displayed suppression of IgE titers, airway inflammation, mucus production, and AHR to ovalbumin 
(OVA) immunization and challenge. In the present study, we developed a new mIL-13 derivative vaccine that contained a keyhole limpet hemocyanin (KLH)-mIL-13 heterocomplex (hereafter named 'KLH-P') without biological activity.

Asthma etiopathogenesis involves genetic susceptibility, environmental factors, and clinical manifestations that are closely associated with the presence of activated Th2 cytokine (IL-4, IL-5 and IL-13) (10). Numerous studies have provided compelling evidence that IL-13 was necessary and sufficient to induce all features of allergic asthma (11), and a therapeutic target for IL-13 is effective. KLH is a xenoantigen largely used in vivo as a vaccine component with optimal carrier qualities, especially for cancer vaccines (12). With the use of KLH, a potent carrier, haptens and peptides would further augment the immunogenicity in conjugate vaccines. This suggests that the critical role of these conjugate vaccines may be the induction of higher levels of T-cell immunity against KLH, which leads to higher levels of $\mathrm{Ab}$ against the conjugated antigens. Based on these findings, we constructed KLH-P by coupling mIL-13 peptide to KLH by aldehyde treatment. In the heterocomplex, each KLH carrier molecule is linked to a large number of mIL-13 peptides by covalent and noncovalent bonds (13), which is devoid of the biological activity of native IL-13 and is still immunogenic in mice.

The novel vaccine could trigger a $B$ cell response and generate $\mathrm{Ab}$ to $\mathrm{mIL}-13$. To our knowledge, we have demonstrated for the first time that KLH-P is able to induce high-titer IL-13 specific IgG with aluminum hydroxide as an adjuvant and prevent mice from airway inflammation, epithelial cell proliferation with goblet cell hyperplasia as well as other features of allergic disease in a mouse model of acute asthma. Most importantly, this study also showed that KLH-P could be a promising vaccine against asthma.

\section{Materials and methods}

Reagents. A mouse IL-13 peptide was selected to generate a new vaccine as previously reported (9). KLH and mIL-13 peptide were purchased from China Peptides Co., Ltd.; mouse IL-13 protein from Invitrogen Life Technologies (Carlsbad, CA, USA); goat anti-mouse IgG and rabbit anti-mouse IgE were from Sigma-Aldrich (Schnelldorf, Germany); the mIL-13 enzyme-linked immunosorbent assay (ELISA) kit, the mIL-4 ELISA kit and the mIFN- $\gamma$ ELISA kit were from R\&D Systems (Minneapolis, MN, USA); the total IgE ELISA kit from MD Biosciences, Inc. (St. Paul, MN, USA); complete and incomplete Freund's adjuvant were from Sigma-Aldrich; OVA was from Sigma-Aldrich; and aluminum hydroxide was from Pierce Chemical Co. (Rockford, IL, USA).

Immunogenicity. The artificial antigen of IL-13 was prepared by complexing mIL-13 with KLH by aldehyde treatment, as previously reported (14). A control was prepared by native KLH sample treated similarly and referenced in the text as KLH. Three New Zealand white rabbits were subcutaneously immunized with conjugates (KLH-P) and developed satisfactory $\mathrm{Ab}$ titers, with the highest titer being $10^{5}$ (data not shown).

Mice. Specific pathogen-free female BALB/c mice (7-8-weekold) were purchased from Beijing Hua Fu Kang Biological
Technology Co., Ltd. (Beijing, China). All protocols used were approved by the Institutional Animal Care and Use Committee of Sichuan University (Chengdu, China).

Protocol of immunization, sensitization, and challenge. Mice were subsequently divided into four groups (Fig. 1) including: i) KLH-P $10 \mu \mathrm{g}(\mathrm{n}=15)$, immunized subcutaneously with $10 \mu \mathrm{g}$ vaccine, then subjected to intraperitoneal sensitization and intranasal challenge with OVA; ii) KLH-P $50 \mu \mathrm{g}(\mathrm{n}=15)$, immunized with $50 \mu \mathrm{g}$ vaccine and sensitized/challenged with OVA; iii) KLH ( $\mathrm{n}=15)$, injected with KLH and sensitized/challenged with OVA; iv) saline $(n=15)$, injected with saline and no exposure to OVA. In this protocol, mice were immunized subcutaneously with vaccine on Weeks 0, 2 and 4 using aluminum hydroxide as an adjuvant, and sensitized on Weeks 5 and 7, by intraperitoneal injection of $20 \mu \mathrm{g}$ OVA emulsified in $2 \mathrm{mg}$ of aluminum hydroxide in a total volume of $200 \mu \mathrm{l}$. After the initial sensitization, the saline group of mice was only exposed to the inhalation of a saline aerosol and three other groups were exposed to an aerosol of $1 \%(\mathrm{w} / \mathrm{v})$ OVA in saline using an ultrasonic nebulizer (Pari-Boy; PariWerke, Starnberg, Germany) for $30 \mathrm{~min}$ for Week 8.

Collection of blood and bronchoalveolar lavage fluid $(B A L F)$ samples. Blood samples were collected on Weeks 1, 3, 5 and 7 before the succeeding OVA administration, and at the endpoint of the experiment. Seventy-two hours after the last challenge, all mice were sacrificed and their blood samples were collected. The samples were centrifuged at $4^{\circ} \mathrm{C}$ for $10 \mathrm{~min}(1,200 \mathrm{rpm})$. Serum was frozen and stored at $-80^{\circ} \mathrm{C}$ for the measurement of cytokines and antibodies by ELISA. BALF was collected as described previously (15). Briefly, after the mice were sacrificed, the chest cavity was exposed, the trachea was carefully intubated with a cannula which was secured with ligatures, $1 \mathrm{ml}$ of cold normal saline was slowly infused into the lungs and withdrawn. The BALF was centrifuged at $4^{\circ} \mathrm{C}$ for $10 \mathrm{~min}(1,200 \mathrm{rpm})$. Supernatants of BALF were collected and stored at $-80^{\circ} \mathrm{C}$ until cytokine and leukotriene measurements were obtained. Serum IL-13specific IgG, total IgE, OVA-specific IgE, and cytokine levels in BALF supernatants were assayed by an ELISA assay following the manufacturer's instructions.

Eosinophil cell counts in BALF. An eosinophil cell count in BALF was performed automatically at the Chengdu GLP Center using an ADVIA2120 automated hematology analyzer (Siemens Healthcare Diagnostics, Inc., Tarrytown, NY, USA).

ELISA. IL-13-specific IgG and OVA-specific IgE levels were measured as described before (16). The levels of IL-13-specific IgG and OVA-specific IgE levels were measured by coating microplates with either mouse IL-13 $(1 \mu \mathrm{g} / \mathrm{ml})$ or OVA $(2 \mu \mathrm{g} / \mathrm{ml})$ at $4^{\circ} \mathrm{C}$ overnight, incubating 2 -fold serially diluted serum samples, flowed by incubation with alkaline phosphatase-conjugated goat anti-mouse IgG for IL-13-specific IgG assay or alkaline phosphatase-conjugated rabbit anti-mouse IgE for OVA-specific IgE assay, and then adding the substrate to develop the reaction. The concentration of IL-13, IL-4 and IFN- $\gamma$ in BALF supernatant were determined using commercial ELISA kits according to the manufacturer's specifications. The concentration of total $\mathrm{IgE}$ in 


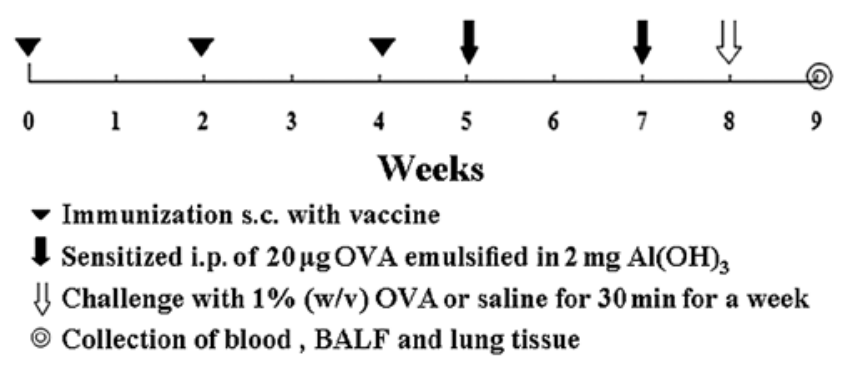

Figure 1. Immunization, sensitization and challenge protocol of mice. The rabbit experiment data is not shown.

the serum was determined using commercial ELISA kits according to the supplier's instructions.

Pulmonary histology. Seventy-two hours after the last challenge, mice were sacrificed and the lungs and trachea were filled with a fixative $(0.8 \%$ formalin, $4 \%$ acetic acid $)$ intratracheally. Lungs were removed and fixed with $10 \%(\mathrm{v} / \mathrm{v})$ neutral-buffered formalin, then dehydrated and embedded in paraffin. For the histological examination, $5-\mu \mathrm{m}$ sections of fixed embedded tissues were cut and placed on glass slides, deparaffinized and stained with hematoxylin and eosin (H\&E) or alcian blue-periodic acid schiff (PAS) and examined using an Olympus IX51 light microscope equipped with a CCD camera. As previously described (15), the degree of peribronchiolar and perivascular inflammation in H\&E stained slides was evaluated on a subjective indexed scale of 0 to 3 : a value of 0 was adjudged when no inflammation was detectable, a value of 1 for occasional perivascular cuffing, a value of 2 for most bronchi or when vessels were surrounded by a thin layer (1 to 5 cells) of inflammatory cells, and a value of 3 when most bronchi or vessels were surrounded by a thick layer ( $>5$ cells) of inflammatory cells. Scores were evaluated by three different observers blinded to the experimental treatment. Glandular hyperplasia was observed using the alcian blue-PAS combined staining in randomly selected bronchi. Alcian blue-PAS stains mucin-secreted cells (goblet cells) and the results were measured as a percentage of alcian blue-PAS positive cells in the total airway epithelia of medium-sized airways. Scoring was performed at a magnification of $x 200$ by examining at least 40 consecutive fields by one observer blinded to experimental data.

Statistical analysis. Each experiment was performed three times with similar results. All images acquired were characteristic of the majority of analyzed tissues. Values were expressed as means \pm standard error of the mean (SEM). The significance of differences between experimental groups was analyzed using one-way ANOVA analysis of variance followed by Newman-Keuls multiple comparison test. Statistical significance was set at $\mathrm{P}<0.05$.

\section{Results}

$K L H-P$ vaccination induction of IL-13-specific IgG. First, we used three New Zealand white rabbits to test the ability of the vaccine (KLH-P) to induce an IL-13-specific IgG response

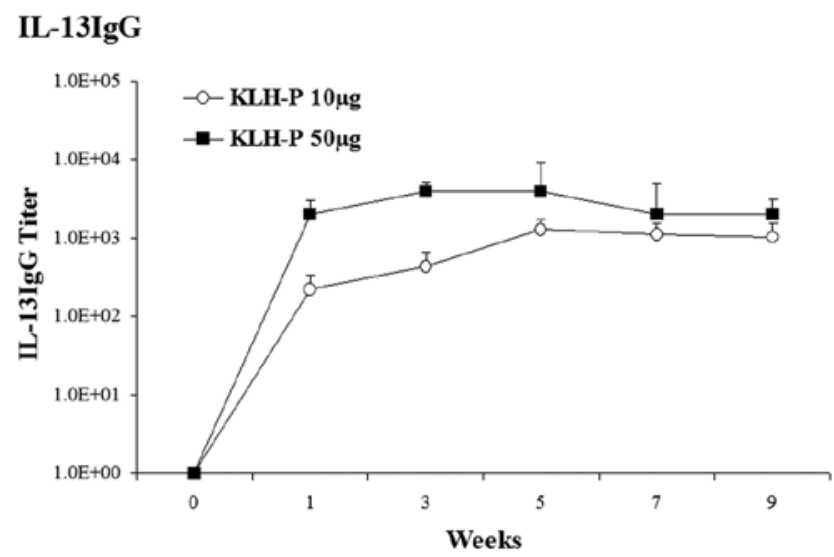

Figure 2. Immunization with KLH-P vaccine induces IL-13-specific IgG in $\mathrm{BALB} / \mathrm{c}$ mice. Mice were immunized using protocol in Fig. 1 subcutaneously with alum as adjuvant. Samples were collected at the time indicated (dose 10 and $50 \mu \mathrm{g}$ ). Serum was 10 -fold dilution series prepared for ELISA. Bars denote standard deviation (SD).

after immunization with the vaccine in the presence of adjuvant (alum or complete/incomplete Freund's adjuvant, CFA/ IFA). All three rabbits immunized with the vaccine developed strong and long-lasting IL-13-specific Ab, which reached high levels (titer up to $10^{5}$, data not shown) and sustained for 1 month before any detectable decrease was measured. Mice were immunized with the vaccine subcutaneously in the presence of an adjuvant (alum) and generated satisfactory $\mathrm{Ab}$ titers. We used two distinct doses to immunize mice to further our knowledge of the immunogenicity of this vaccine. IL-13-specific IgG in serum response was displayed in a dose-dependent manner within the dose range used. The result indicated that $10 \mu \mathrm{g}$ of vaccine given subcutaneously (s.c.) was able to efficiently induce IgG responses (Fig. 2). During the period of the experiment, 2 weeks after sensitization with OVA (Week 7), IL-13-specific IgG titers slightly decreased when compared to those at Week 5 immediately after the second immunization with the vaccine, but remained high until the end of the study. The data demonstrate that the KLH-P vaccine is immunogenic and displays dose-dependent effects using aluminum hydroxide as adjuvant.

Total and OVA-specific IgE are reduced by KLH-P vaccination. To determine whether KLH-P vaccine could modify an OVA-specific T-helper 2 cell (Th2) response in vivo by analyzing circulating IgE Ab levels $72 \mathrm{~h}$ after OVA challenge, sera were collected on Weeks $0,1,3,5,7$ and again at the endpoint of the experiment (Week 9). Both the mean levels of serum total and OVA-specific IgE antibodies in the vaccinetreated groups were markedly reduced compared to those seen in the KLH groups (Fig. 3A and B). Total and OVA-specific IgE levels were greatly increased after intraperitoneal sensitization and intranasal challenge with OVA two times. However, vaccination with KLH-P significantly suppressed total IgE (Week 7: $\mathrm{P}<0.01, \mathrm{P}<0.001$ for KLH-P 10 and $50 \mu \mathrm{g}$, respectively; Week 9: $\mathrm{P}<0.05$ for KLH-P $50 \mu \mathrm{g}$ ) (Fig. 3A). In agreement with the inhibitory effect on total IgE, immunization of KLH-P at $50 \mu \mathrm{g}$ visibly reduced OVA-specific IgE levels compared to the KLH group. After 2 times of sensitization with OVA, even 

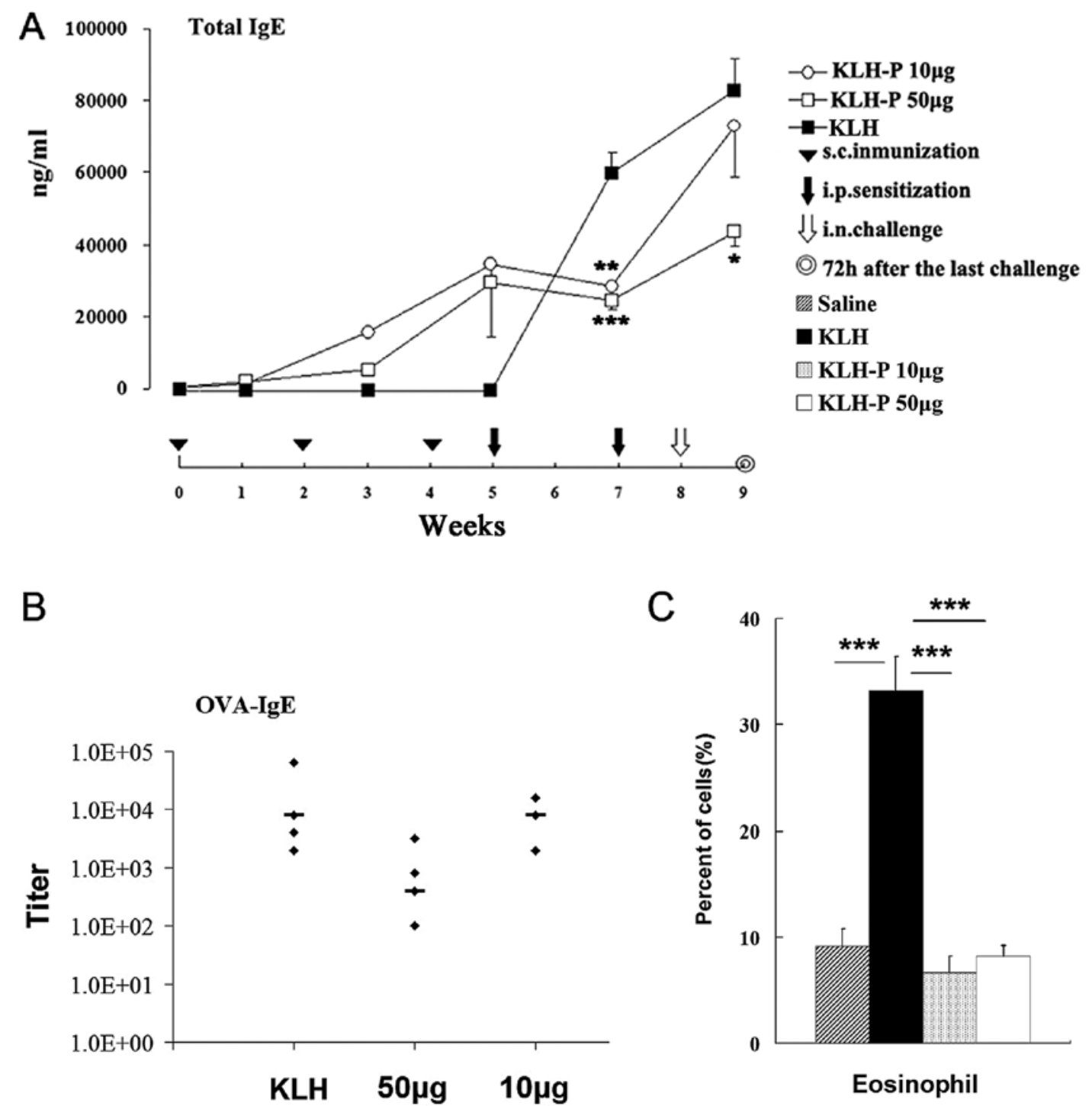

Figure 3. Total and OVA-specific IgE in serum were reduced by KLH-P vaccine. (A) Quantification of total IgE Ab in serum samples collected at the times indicated; the serum was diluted at 1:500. (B) Detection of specific Ab against OVA in serum samples collected at the endpoint of the experiment with 5 single mice in each group. (C) KLH-P vaccine reduces eosinophil percentage in BALF. Data are reported as mean \pm SEM. Significant differences are shown as ${ }^{*} \mathrm{P}<0.05,{ }^{* * *} \mathrm{P}<0.01$ and ${ }^{* * *} \mathrm{P}<0.001$. Bars denote standard error (SE).

though IL-13-specific IgG levels slightly decreased to some degree compared with those at Week 5 , it still remained $10^{3}$ at the endpoint of the study. In this experiment, sera gathered at the endpoint with 5 single mice in each group were analyzed to measure OVA-specific IgE (Week 9) (Fig. 3B). It appeared that the KLH-P vaccine displayed a visible effect in inhibition of OVA-specific IgE which was dose-dependent, although there was no statistical significance observed.

KLH-P vaccine reduces eosinophil percentage in BALF. To determine the contribution of this vaccine to eosinophil recruitment after allergen challenge, BALF was collected $72 \mathrm{~h}$ after the last OVA aerosol challenge, and differential cell counts were detected with an ADVIA2120 automated hematology analyzer. OVA inhalation markedly $(\mathrm{P}<0.001)$ increased the percentages of eosinophils compared with saline control. In contrast, immunization with KLH-P vaccine in either dose significantly $(\mathrm{P}<0.001)$ inhibited eosinophil recruitment to BALF compared to the KLH group (Fig. 3C). These results suggest that KLH-P vaccination suppressed the accumulation of eosinophils in BALF.

IL-13, IL-4 and IFN- $\gamma$ levels in BALF. To assess whether IL-13, IL-4 and IFN- $\gamma$ levels were downregulated by this vaccine, BALF was collected and analyzed with ELISA assays. IL-13 was increased in the BALF from mice exposed to OVA. Most importantly, such an increase was inhibited by a previous vaccination with KLH-P in either dose $(\mathrm{P}<0.001)$ (Fig. 4A). It's worth noting that a different vaccine dose did not significantly affect the mean levels of IL-13 in BALF. The IL-4 (Fig. 4B) precludes any statistical significance despite the appearance of a large decrease. When compared to normal mice exposed only to saline, IFN- $\gamma$ levels were apparently $(\mathrm{P}<0.05)($ Fig. 4C) increased in OVA-sensitized and challenged mice. However, the mean level of IFN- $\gamma$ was not significantly modified in the group treated by vaccine. Taking these results together, except for IL-13, the current vaccine immunization did not produce 

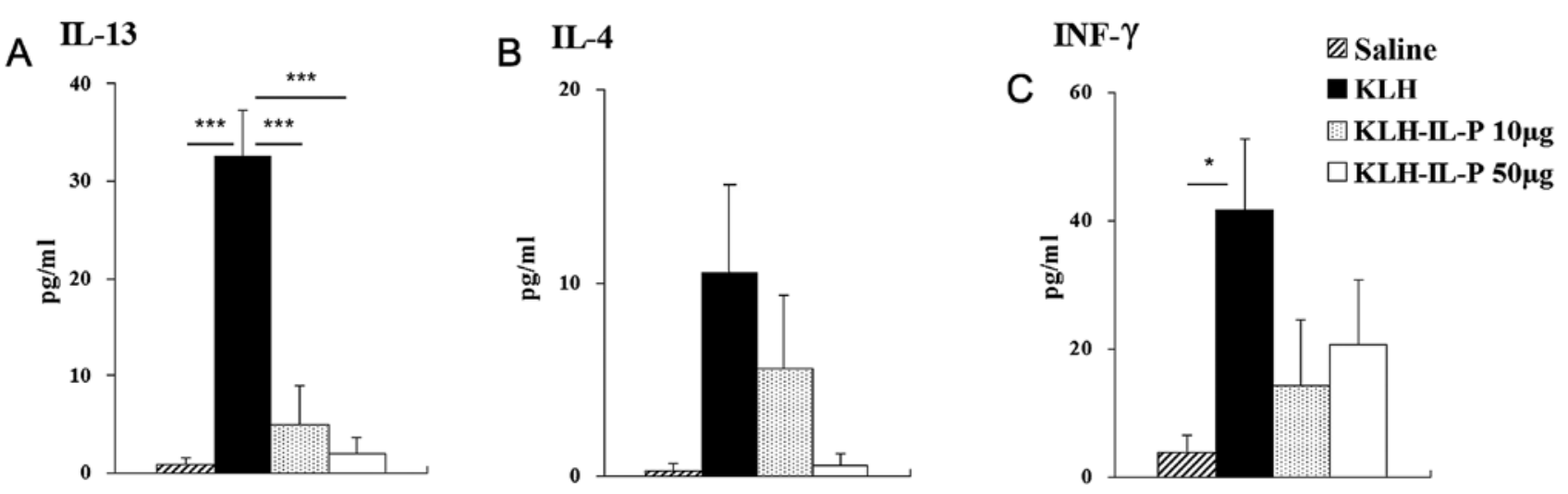

Figure 4. Effects of KLH-P vaccine on cytokine accumulation in BALF. Immunization with KLH-P in either dose inhibited IL-13 accumulation in BALF compared with KLH group. (A) IL-13; (B) IL-4; (C) IFN- $\gamma$; Significant differences are shown as " $\mathrm{P}<0.05$ and ${ }^{* * * *} \mathrm{P}<0.001$. Bars denote standard error (SE).

significant effects on cytokine levels compared with the KLH group.

KLH-P vaccine suppresses OVA-induced tissue leukocyte influx and airway inflammation. To evaluate allergic airways inflammation, lung tissues were collected $72 \mathrm{~h}$ after the last OVA exposure and histological analyses were performed. Consistent with previous BALF cytology, H\&E staining of lung sections confirmed that the accumulation of inflammatory cells in the airways was also suppressed in mice that received KLH-P vaccine (Fig. 5A). Semi-quantitative analyses of tissue inflammation were performed in each group, and a peribronchial inflammation score was evaluated for 5-7 bronchi per mouse (Fig. 5B). There was evidence of significant peribronchial accumulation of inflammatory cells in mice exposed to OVA, as representative examples are presented in Fig. 5A. In contrast, KLH-P vaccinated mice showed substantial attenuation in the eosinophil rich leukocyte infiltration in the peribronchiolar regions. Seventy-two hours after the last OVA challenge, the inflammation scores of peribronchial as well as total lung were increased significantly $(\mathrm{P}<0.001)$ in the KLH group compared with scores in either dose of the KLH-P vaccine-treated group. Additionally, the lung inflammation score observed after immunization with the KLH-P vaccine in $10 \mu \mathrm{g}$ dose is significantly $(\mathrm{P}<0.01)$ lower than that of a group with a $50 \mu \mathrm{g}$ dose. Taken together, these results further confirmed that the KLH-P vaccine significantly suppressed allergen-induced leukocyte influx and airway inflammation.

The KLH-P vaccine suppresses airway goblet cell metaplasia and mucus hypersecretion. Airway epithelial cell hyperplasia and mucus secretion were assessed by an alcian blue-PAS combined staining. In normal mice, goblet cells comprise less than $20 \%$ of cells in the bronchial epithelium, and the number of goblet cells was greatly decreased in the segmental bronchus (17). However, we observed an increased number of goblet cells in the segmental bronchus, and prominent mucus production by the airway epithelium in the KLH group compared to the KLH-P vaccine-treated groups (Fig. 6A). We performed semi-quantitative analysis of goblet cells in each group (Fig. 6B). Goblet cell metaplasia and mucin hypersecretion were dramatically $(\mathrm{P}<0.001)$ inhibited in the KLH-P vaccine treated groups. There was no significant difference between the high-dose and low-dose groups $(\mathrm{P}>0.05)$. PAS shows the red-purple cytoplasmic inclusion (neutral mucus) and alcian blue shows the blue cytoplasmic inclusion (acid mucus). Naturally, the same group samples or a single slice showed different intensity staining neutral mucopolysaccharide (NMPS) and acid mucopolysaccharide (AMPS). There is almost no neutral or acid mucus production in the epithelial cell in saline control and vaccinated mice compared a hypersecretion of acid mucus from goblet cells in the KLH group. As a result, our findings have shown that the KLH-P vaccine effectively suppressed allergen-induced airway goblet cell metaplasia and mucus hypersecretion.

\section{Discussion}

Asthma is a lifelong disease that causes wheezing, breathlessness, chest tightness, and coughing. It can limit a person's quality of life. The control of asthma symptomatology requires a complex treatment plan that changes according to presenting symptoms. Although most people with asthma may control their symptoms and prevent asthma attacks by using medication, such as inhaled corticosteroids. Recent studies have demonstrated that adherence rates for asthmatic patients are particularly problematic. Barriers to treatment include prolonged and complex regimens, adverse effects, cost, and delayed onset of action. Less than half of the patients could adhere to their prescribed regimens $(18,19)$, contributing to the poor clinical outcomes in the treatment of asthma. Taking into account the barriers of treatment-related therapy, a novel effective treatment of asthma is strongly needed. In the present study, we aimed to develop a novel vaccine to improve the efficacy and safety of therapeutic strategies. The KLH-P vaccine has been constructed and applied to asthmatic BALB/c mice sensitized and challenged with OVA. We reported here that immunization of mice with this murine KLH-P-induced polyclonal autoantibody neutralized excessive mIL-13 bioactivity and further inhibited the symptoms of experimental model of murine asthma.

Immunization with our mIL-13 peptide kinoid induces neutralizing auto-antibodies to mIL-13. We used two distinct doses to further our knowledge of the immunogenicity of this vaccine. The results indicate that just $10 \mu \mathrm{g}$ of vaccine given s.c., rather than $100 \mu \mathrm{g}$ given previously, was able to 
A 1
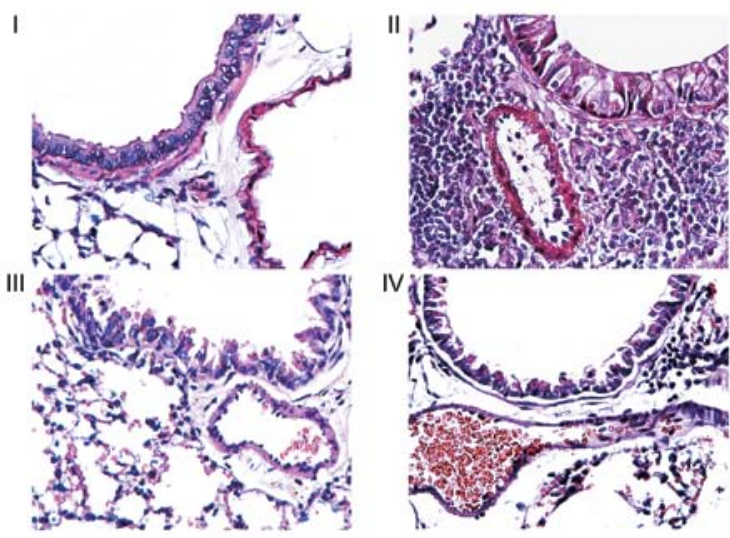

B

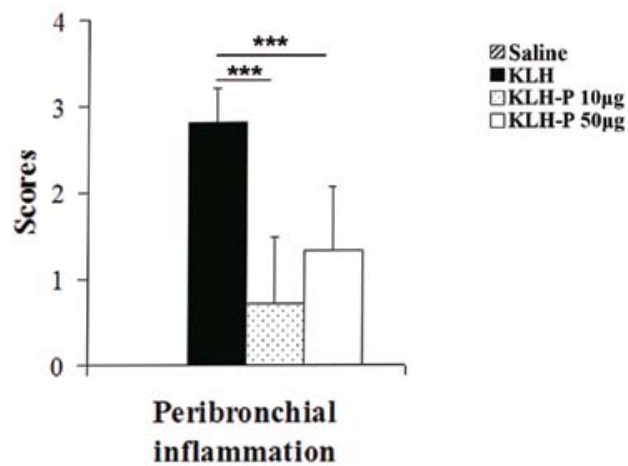

Figure 5. Airway tissue inflammation was significantly decreased by KLH-P vaccine analyzed by H\&E stain. (A) Representative photomicrographs are shown. (I) Saline, injected with saline and no exposure to OVA; (II) KLH, vaccinated with KLH and then subjected to intraperitoneal sensitization and intranasal challenge with OVA; (III and IV) KLH-P, vaccinated at a lowdose $(10 \mu \mathrm{g})$ and a high-dose $(50 \mu \mathrm{g})$ respectively then sensitized/challenged with OVA significantly reduces the inflammation scores of peribronchial as well as total lung $(\mathrm{P}<0.001)$. (B) Semi-quantification analysis was performed to assess histological changes ( $\mathrm{n}=5 / 15$ group). The noted values represent mean \pm SD on a minimum of 5-7 bronchi per mouse for 5 animals a group. Significant differences were shown as ${ }^{* * *} \mathrm{P}<0.001$. Bars denote standard error (SE).

efficiently induce IgG responses (Fig. 2). Although there was a slight decrease of IL-13-specific IgG titers at Week 7 compared with those at Week 5, features of airway allergic inflammatory response were prevented as long as the transient serum remained above an effective threshold. The rapid decline of neutralizing Abs after kinoid immunization (Fig. 2) may be due to the short life of B cell memory in the absence of specific T cell help (20). Using the murine model of allergic asthma and based on a preventative design, we further assessed the efficacy of our IL-13 peptides kinoid vaccine in preventing total and OVA-specific IgE amounts in serum, pulmonary eosinophilia, allergic airway inflammation, and airway goblet cell hyperplasia and mucus secretion.

IgE plays an important role in triggering the allergic cascade, and anti-IgE therapy has confirmed its central role in the inflammatory process of allergic asthma (21). Studies have demonstrated that IL-13 contributes to IgE synthesis (22). Our results demonstrated that vaccination with IL-13 peptides kiniod vaccine suppressed allergen-induced total and OVA-specific IgE in a dose-dependent manner. This is significant in order to prevent allergic symptoms. Reduction
A
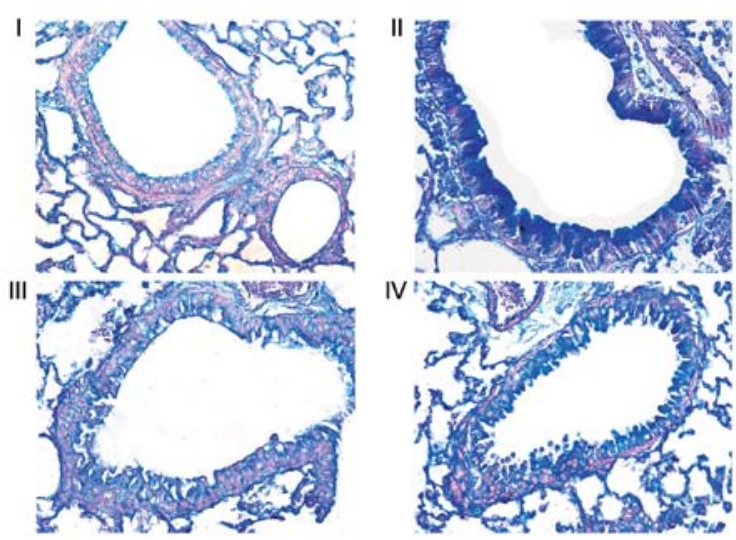

B

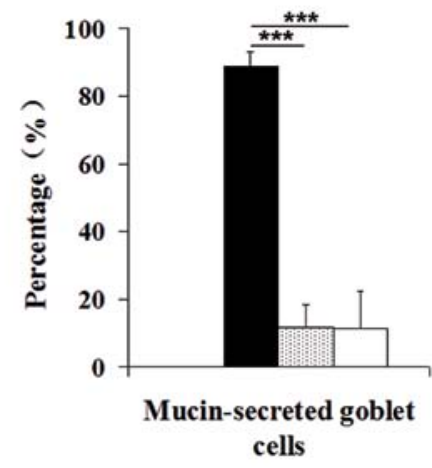

Q Saline

- KLH

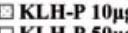

Figure 6. Airway goblet cell hyperplasia and mucus hypersecretion was notably decreased by KLH-P vaccine analyzed following alcian blue-PAS stain. (A) Representative photomicrographs are shown. Neutral and acidic mucus are overproduced in goblet cells of segmental bronchus. PAS and alcian blue stain, PAS showing the red-purple cytoplasmic inclusion (neutral mucus) and alcian blue showing the blue cytoplasmic inclusion (acid mucus), x200 showing lung sections from (I-IV). (I) Saline, injected with saline and no exposure to OVA; (II) KLH, vaccinated with KLH and then subjected to intraperitoneal sensitization and intranasal challenge with OVA; (III and IV) KLH-P, vaccinated at a low-dose $(10 \mu \mathrm{g})$ and a high-dose $(50 \mu \mathrm{g})$ respectively then sensitized/challenged with OVA significantly reduces airway mucin-secreted goblet cell metaplasia and mucus hypersecretion $(\mathrm{P}<0.001)$. There is almost no neutral or acid mucus production in the epithelial cell in saline group and decreased mucus production and fewer goblet cells are seen in either dose-treated mice compared to hypersecretion of acid mucus in the KLH-treated group. (B) Semi-quantification analysis was performed to assess airway epithelium histological changes ( $n=5 / 15$ group). The noted values represent the mean $\pm \mathrm{SD}$ on a minimum of 5-7 bronchi per mouse for five animals per group. Significant differences were shown as ${ }^{* * *} \mathrm{P}<0.001$. Bars denote standard error (SE).

in $\operatorname{IgE}$ levels leads to reduction in both basophil and mast cell mediator response to allergen, and causes a decrease in allergic inflammation by reducing the high-affinity $\operatorname{IgE}$ receptor on mast cells and dendritic cells $(23,24)$. Therefore, the suppression of $\mathrm{Th} 2$ responses IgE levels and eventually asthmatic symptoms may be achieved through downregulation of IL-13. Collectively, the effective suppression of total and OVA-specific IgE suggested our IL-13 peptides kiniod vaccine has a strong potential in this regard.

Airway eosinophilia is considered a cardinal feature of allergic asthma (25). It is thought that eosinophils contribute substantially not only to airway remodeling (26), but also to acute responses, such as goblet cell hyperplasia (27). As eosinophilia in the airway is an important indicator of asthma, we performed eosinophil cell counts in BALF and histo- 
pathological examination to assess the efficacy of our IL-13 peptides kinoid vaccine on airway inflammation and goblet cell hyperplasia. The results showed that immunization with two different doses (10 or $50 \mu \mathrm{g}$ ) significantly suppressed the accumulation of eosinophils in BALF ( $\mathrm{P}<0.001$ for both).

Furthermore, after immunization with the KLH-P vaccine, we observed a dramatic suppression of allergen-induced leukocyte influx, airway inflammation, and mucus production. Mucus plugging has been recognized as a major factor contributing to airway obstruction and mortality associated with acute severe asthma (28-30). The continuity of surface goblet cell granules and intraluminal mucus are an important source of the secretions and contribute to the formation of mucus plugging in asthma $(30,31)$. It appears that mucus hypersecretion is related to the pathogenesis of acute asthma (30), and abnormal proliferation of goblet cells is necessary for hypersecretion $(31,32)$. The fact that acute airway administration of IL-13 $(32,33)$ or overproduction of IL13 in the lung (34) could rapidly induce mucus cell hyperplasia in nonimmunized mice and the formation of Charcot-Leyden crystals (35) indicates that IL-13 may play a role in promoting goblet cell hyperplasia and mucus production. Further studies demonstrated that IL-13 gene knockout (36) or blockade of IL-13 by either administration of the soluble IL-13 $\alpha 2$-Ig $(1,4)$ or through IL-13 gene targeting in mice (36) prevents mucus hypersecretion, and can reverse established goblet cell metaplasia. Consistent with these observations, after immunization with the KLH-P vaccine, we observed a dramatic suppression of allergen-induced leukocyte influx, airway inflammation, and mucus production, which is indicated by mucin-secreted goblet cells after allergen challenge.

The mIL-13 kinoid immunization is safe. There were no adverse reactions to the kinoid preparation. The lack of autoimmune pathology due to the absence of $\mathrm{T}$ cell response after other anti-cytokine immunizations was demonstrated in kinoid-immunized mice, including TNF (37), VEGF (14), IFN- $\alpha$ (38), or other kinoids, minimizing the risk of iatrogenic complications after kinoids immunization. Normal development, a normal growth curve, and a lack of adverse clinical features in kinoid-immunized mice compared to controls suggest that this may represent an alternative strategy to passive anti-IL-13 mAb therapy or drugs that block IL-13 activity currently used in patients.

Indeed, in our study anti-mIL-13 Abs did not substitute for, but acted rather as an adjunct treatment in addition to conventional treatments, as recommended in clinical protocols for allergic asthma treatment. Particularly, after its validation in humans, the vaccine could represent an alternative prevention for patients with seasonal asthma. Furthermore, to maintain an effective Ab threshold, the frequency of kinoid boosters should be optimally determined by monitoring levels of circulating Abs, maintaining the logic of metronomic scheduled treatments (39).

Compared with passive immunization therapy, active immunization with the kinoid vaccine described here would have the following advantages. First, the generation of polyclonal antibodies by active immunization excludes the formation of anti-idiotypic antibodies, limiting the rate of therapeutic failure. Second, the low frequency of boost immunization would foster patient compliance. Finally, the proposed therapeutic approach would represent a low cost.
In conclusion, our findings demonstrate that the administration of IL-13 peptide kinoid vaccines triggers a strong and transient $\mathrm{Ab}$ response to IL-13, which prevents the pathogenic effects of this cytokine in an experimental murine model of acute asthma induced by OVA. This may become an effective therapeutic approach to allergic asthma treatment and may provide a new therapeutic strategy for treating or vaccinating against other diseases where overproduction of cytokines play a critical role in pathogenesis.

\section{Acknowledgements}

This study was supported by the Program for New Century Excellent Talents in University (NCET) (NCET-09-0575) and the National Natural Science Foundation of China (Grant no. 30973453).

\section{References}

1. Wills-Karp M, Luyimbazi J, Xu X, Schofield B, et al: Interleukin-13: central mediator of allergic asthma. Science 282: 2258-2261, 1998 .

2. Finkelman FD, Hogan SP, Hershey GK, Rothenberg ME and Wills-Karp M: Importance of cytokines in murine allergic airway disease and human asthma. J Immunol 184: 1663-1674, 2010.

3. Hamid Q and Tulic M: Immunobiology of asthma. Annu Rev Physiol 71: 489-507, 2009.

4. Grünig G, Warnock M, Wakil AE, et al: Requirement for IL-13 independently of IL-4 in experimental asthma. Science 282: 2261-2263, 1998.

5. Yang G, Li L, Volk A, et al: Therapeutic dosing with antiinterleukin-13 monoclonal antibody inhibits asthma progression in mice. J Pharmacol Exp Ther 313: 8-15, 2005.

6. Kasaian MT and Miller DK: IL-13 as a therapeutic target for respiratory disease. Biochem Pharmacol 76: 147-155, 2008.

7. Holgate S, Chuchalin A, Hebert J, et al: Efficacy and safety of a recombinant anti-immunoglobulin $\mathrm{E}$ antibody (omalizumab) in severe allergic asthma. Clin Exp Allergy 34: 632-638, 2004.

8. Sandborn WJ: New concepts in anti-tumor necrosis factor therapy for inflammatory bowel disease. Rev Gastroenterol Disord 5: 10-18, 2005.

9. Ma Y, HayGlass KT, Becker AB, et al: Novel recombinant interleukin-13 peptide-based vaccine reduces airway allergic inflammatory responses in mice. Am J Respir Crit Care Med 176: 439-445, 2007.

10. Wills-Karp M: Immunologic basis of antigen-induced airway hyperresponsiveness. Annu Rev Immunol 17: 255-281, 1999.

11. Wills-Karp M: Interleukin-13 in asthma pathogenesis. Immunol Rev 202: 175-190, 2004.

12. Sabbatini PJ, Ragupathi G, Hood C, et al: Pilot study of a heptavalent vaccine-keyhole limpet hemocyanin conjugate plus QS21 in patients with epithelial ovarian, fallopian tube, or peritoneal cancer. Clin Cancer Res 13: 4170-4177, 2007.

13. Le Buanec $H$ and Zagury D: Stable immunogenic product comprising antigenic heterocomplexes. US Patent 7972603. Filed Septemper 16, 2003; issued July 5, 2011.

14. Rad FH, Le Buanec H, Paturance S, et al: VEGF kinoid vaccine, a therapeutic approach against tumor angiogenesis and metastases. Proc Natl Acad Sci USA 104: 2837-2842, 2007.

15. Kwak YG, Song CH, Yi HK, et al: Involvement of PTEN in airway hyperresponsiveness and inflammation in bronchial asthma. J Clin Investig 111: 1083-1092, 2003.

16. Ma Y, Ma AG and Peng Z: A potential immunotherapy approach: mucosal immunization with an IL-13 peptide-based virus-like particle vaccine in a mouse asthma model. Vaccine 25: 8091-8099, 2007.

17. Ma JY, Medicherla S, Kerr I, Mangadu R, Protter AA and Higgins LS: Selective p38- $\alpha$ mitogen-activated protein kinase inhibitor attenuates lung inflammation and fibrosis in IL-13 transgenic mouse model of asthma. J Asthma Allergy 1: 31-44, 2008.

18. Milgrom H, Bender B, Ackerson L, Bowry P, Smith B and Rand C: Noncompliance and treatment failure in children with asthma. J Allergy Clin Immunol 98: 1051-1057, 1996. 
19. Rand C, Wise R, Nides M, et al: Metered-dose inhaler adherence in a clinical trial. Am Rev Respir Dis 146: 1559-1564, 1992.

20. Gray D and Skarvall H: B-cell memory is short-lived in the absence of antigen. Nature 336: 70-73, 1998.

21. Holgate S, Casale T, Wenzel S, Bousquet J, Deniz Y and Reisner C: The anti-inflammatory effects of omalizumab confirm the central role of $\mathrm{IgE}$ in allergic inflammation. J Allergy Clin Immunol 115: 459-465, 2005.

22. Emson CL, Bell SE, Jones A, Wisden W and McKenzie AN: Interleukin (IL)-4-independent induction of immunoglobulin (Ig) E, and perturbation of T cell development in transgenic mice expressing IL-13. J Exp Med 188: 399-404, 1998.

23. Beck LA, Marcotte GV, MacGlashan D, Togias A and Saini S: Omalizumab-induced reductions in mast cell FceRI expression and function. J Allergy Clin Immunol 114: 527-530, 2004.

24. Prussin C, Griffith DT, Boesel KM, Lin H, Foster B and Casale TB: Omalizumab treatment downregulates dendritic cell FceRI expression. J Allergy Clin Immunol 112: 1147-1154, 2003.

25. Epstein MM: Do mouse models of allergic asthma mimic clinical disease? Int Arch Allergy Immunol 133: 84-100, 2000.

26. Lee JJ, Dimina D, Macias MP, et al: Defining a link with asthma in mice congenitally deficient in eosinophils. Science 305 : 1773-1776, 2004.

27. Dabbagh K, Takeyama K, Lee HM, Ueki IF, Lausier JA and Nadel JA: IL-4 induces mucin gene expression and goblet cell metaplasia in vitro and in vivo. J Immunol 162: 6233-6237, 1999.

28. Agusti C, Takeyama K, Cardell LO, et al: Goblet cell degranulation after antigen challenge in sensitized guinea pigs. Role of neutrophils. Am J Respir Crit Care Med 158: 1253-1258, 1998.

29. Dunnill M: The pathology of asthma, with special reference to changes in the bronchial mucosa. J Clin Pathol 13: 27-33, 1960.

30. Shimura S, Andoh Y, Haraguchi M and Shirato K: Continuity of airway goblet cells and intraluminal mucus in the airways of patients with bronchial asthma. Eur Respir J 9: 1395-1401, 1996.
31. Aikawa T, Shimura S, Sasaki H, Ebina M and Takishima T: Marked goblet cell hyperplasia with mucus accumulation in the airways of patients who died of severe acute asthma attack. Chest 101: 916-921 1992.

32. Nakano T, Inoue H, Fukuyama S, et al: Niflumic acid suppresses interleukin-13-induced asthma phenotypes. Am J Respir Crit Care Med 173: 1216-1221, 2006.

33. Kibe A, Inoue H, Fukuyama S, et al: Differential regulation by glucocorticoid of interleukin-13-induced eosinophilia, hyperresponsiveness, and goblet cell hyperplasia in mouse airways. Am J Respir Crit Care Med 167: 50-56, 2003.

34. Fulkerson PC, Fischetti CA, Hassman LM, Nikolaidis NM and Rothenberg ME: Persistent effects induced by IL-13 in the lung. Am J Respir Cell Mol Biol 35: 337-346, 2006.

35. Zhu Z, Homer RJ, Wang Z, et al: Pulmonary expression of interleukin-13 causes inflammation, mucus hypersecretion, subepithelial fibrosis, physiologic abnormalities, and eotaxin production. J Clin Investig 103: 779-788, 1999.

36. Walter DM, McIntire JJ, Berry G, et al: Critical role for IL-13 in the development of allergen-induced airway hyperreactivity. J Immunol 167: 4668-4675, 2001.

37. Le Buanec H, Delavallée L, Bessis N, et al: TNFa kinoid vaccination-induced neutralizing antibodies to $\mathrm{TNF} \alpha$ protect mice from autologous TNF $\alpha$-driven chronic and acute inflammation. Proc Natl Acad Sci USA 103: 19442-19447, 2006.

38. Zagury D, Le Buanec H, Mathian A, et al: IFN $\alpha$ kinoid vaccineinduced neutralizing antibodies prevent clinical manifestations in a lupus flare murine model. Proc Natl Acad Sci USA 106: 5294-5299, 2009

39. Kerbel RS and Kamen BA: The anti-angiogenic basis of metronomic chemotherapy. Nat Rev Cancer 4: 423-436, 2004. 